\title{
Complicated Bilateral Pulmonary Hydatid Cysts
}

\author{
Dharmendra Joshi ${ }^{1 *}$, MD Abir Tazim Chowdhury ${ }^{1}$, Manmohan Bir Shrestha ${ }^{2}$, Hossain Al Mahmud ${ }^{3}$, \\ Serajus Salekin ${ }^{3}$, MD Osman Gonni ${ }^{3}$ and MD Kamrul Alam ${ }^{3}$
}

\author{
${ }^{1}$ Department of Cardiovascular and Thoracic Surgery, Bangabandhu Sheikh Mujib Medical University, Bangladesh \\ ${ }^{2}$ Department of Radiology, Bangabandhu Sheikh Mujib Medical University, Bangladesh \\ ${ }^{3}$ Department of Thoracic Surgery, Dhaka Medical College and Hospital, Bangladesh
}

\begin{abstract}
Hydatid disease also known as hydatidosis is an endemic parasitic zoonotic disease in some parts of world including Mediterranean basin, South America and Australia, caused by Echinococcus granulosus. Liver is the commonest site for hydatid disease followed by the lungs. The disease is not uncommon although human beings are accidental intermediate hosts in the natural history of hydatidosis but bilateral pulmonary hydatidosis is rare. Patient may be asymptomatic or occasionally present with fever, chest pain, cough and hemoptysis. Ruptured pulmonary hydatid cyst may lead to life threatening complication i.e. anaphylaxis. The hydatid serology results are often negative in pulmonary hydatid disease. Chest X-ray and CT scan of chest may be preoperative diagnostic investigations. Surgical intervention plays gold standard role for the treatment of pulmonary hydatidosis. We are reporting a rare case of complicated bilateral pulmonary hydatid cysts with its operative management and outcome.
\end{abstract}

\section{Keywords}

Bilateral pulmonary hydatid cysts, Echinococcosis, Hydatidosis, Lung, CT Scan

\section{Introduction}

Human echinococcosis is a well-known parasitic zoonotic disease with worldwide distribution which is caused by tapeworms of the genus Echinococcus [1]. Echinococcus has four species, human infestations in majority of cases are caused by E. granulosus [2]. The life-cycle of hydatid tapeworm completes in two hosts where the definitive hosts are canine animals like dogs and warm-blooded vertebrates such as sheep, cattle, pigs, goats and horses are the intermediate hosts. Humans are considered as accidental intermediate hosts and harbor cysts. So, two mammalian species are required for completion of the life cycle of hydatid tapeworm, but the man being dead end host, direct transmission of echinococcus from human to human does not occur [3]. E. granulosus is most commonly found in liver (55-70\%) followed by lungs (18-35\%) in adult.

However, the lungs are most common site of infestation by $E$. granulosis in children. In about $10 \%$ of the cases, other sites like spleen, kidney, brain, bone etc. may be involved. The liver and lungs can be affected simultaneously in about $5-13 \%$ of the cases and about $20 \%$ of all pulmonary hydatid cyst may be bilateral $[2,4]$.

The pulmonary hydatid cyst may range from $1 \mathrm{~cm}$ to 15 $\mathrm{cm}$ in size. Patients with pulmonary hydatidosis may be asymptomatic. However, patients may present with chronic cough, expectoration, breathlessness, hemoptysis, chest pain, pneumothorax, pulmonary embolism and eosinophilic pneumonitis. It may be complicated by rupture of the cyst into bronchial tree and results bronchospasm, symptoms of anaphylaxis, fever, hydropneumothorax, pyothorax with pleural hydatidosis. Incidence of per-operative complication during surgery of pulmonary hydatid cyst is about $1 / 5000$ to $1 / 20000$ procedures and mortality rate is about 3-6\% [5].

In this study, we report a case of complicated bilateral pulmonary hydatid cysts with ruptured and infected hydatid cyst of left lung along with its management and discussion of the related literatures.

\section{Case Report}

A 42-years-old, housewife, non-smoker, non-asthmatic, non-diabetic and hypertensive patient had productive cough and right sided chest pain about 2-years back. She was diag-

*Corresponding author: Dharmendra Joshi, Department of Cardiovascular and Thoracic Surgery, Bangabandhu Sheikh Mujib Medical University, Bangladesh, Tel: +8801720378081

Accepted: March 29, 2020

Published online: March 31, 2020

Citation: Joshi D, Chowdhury AT, Shrestha MB, et al. (2020) Complicated Bilateral Pulmonary Hydatid Cysts. J Cardiothorac Surg Ther 4(1):52-56 


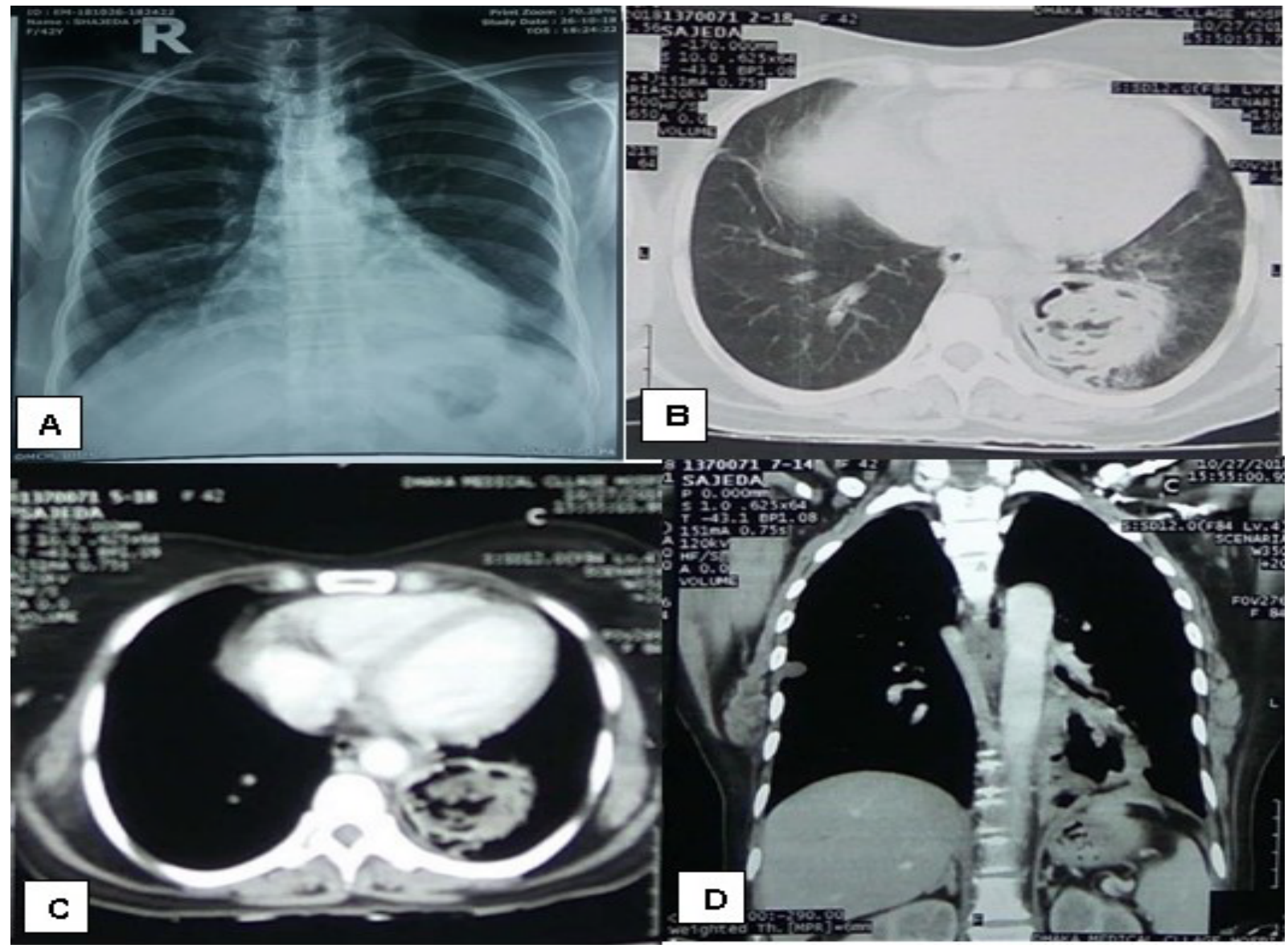

Figure 1: Chest X-ray (P/A view) A) Computed Tomography (CT) scans of chest of the patient before the second operation, Axial slices; B) Pulmonary window view; C) I/V Contrast Mediastinal view and D) Coronal slice showing pulmonary hydatidosis (left).

nosed clinico-radiologically as a case of bilateral pulmonary hydatid cysts. She underwent staged VATS enucleation of right pulmonary hydatid cyst at Dhaka Medical College and Hospital, Bangladesh on 22/02/2017. Her symptoms subsided after the operation and she did not follow the advice for second operation after 3 months due to her personal affairs.

Now she presented on 24/10/2018 with the complaint of cough with foul-smelling "grape skin" like production for last 22 days. She has been suffering from low grade fever occasionally but undocumented and without night sweating, negative history of Pulmonary Tuberculosis (TB) or any contact with patient of TB. She also complained of pain in all over her chest, more in left anterior side, not radiating to other site of body and without any aggravating and relieving factors.

On examination, the patient was mildly anemic and her height was $140 \mathrm{~cm}$, weight: $41 \mathrm{~kg}$, pulse: $80 \mathrm{bpm}$, regular, blood pressure: $120 / 70 \mathrm{mmHg}$, respiratory rate: 12 breaths/ min. Her systemic examination reveals normal findings except respiratory system examination showing multiple small scar marks of previous surgery on right side of the chest, increased tactile vocal fremitus, dull percussion notes and bronchial breath sound in $7^{\text {th }}, 8^{\text {th }} \& 9^{\text {th }}$ inter costal spaces along left dorsal scapular line as well as fine crepitation in left lung field, both during inspiration and expiration, not associated with cough.
Further laboratory evaluation revealed normal Complete Blood Count except Hemoglobin: $9.9 \mathrm{gm} / \mathrm{dL}$, Eosinophils count: $7 \%$ and Absolute Eosinophil Count: 559. Spirometry showed small airway obstruction with FEV1/FVC: $80.3 \%$. Chest $\mathrm{X}$-ray $\mathrm{P} / \mathrm{A}$ view (Figure 1 ) revealed ill-defined opacities in right lower zone. Left side was apparently normal. Chest CT scans (Figure 1) showed a cavitary lesion having thick asymmetric wall and air fluid density was noted at posterior basal segment of left lower lobe. After I/V contrast, wall enhancement was seen, suggesting Pulmonary Hydatid Cyst (Left).

We reviewed her previous investigations done before the first operation. Eosinophils count was normal and Echinococcus Antibody was negative. Chest X-ray P/A view (A) and right lateral view (B) (Figure 2) showed a large round soft tissue opacity with well-defined margin in right lower zone which obscure the right heart border. Another large well-defined soft tissue opacity was noted in left lower zone which could be seen through the heart. Chest CT scans (Figure 2) had revealed a large thick-walled hypodense lesion having smooth inner and outer margin of the wall in right middle lobe. Similar characteristic lesion was also noted in posterior basal segment of left lower lobe. After I/V contrast, enhancement of the wall of the lesions was noted. It suggested Bilateral Pulmonary Hydatid Cysts. Later it was confirmed by histopathology report. 


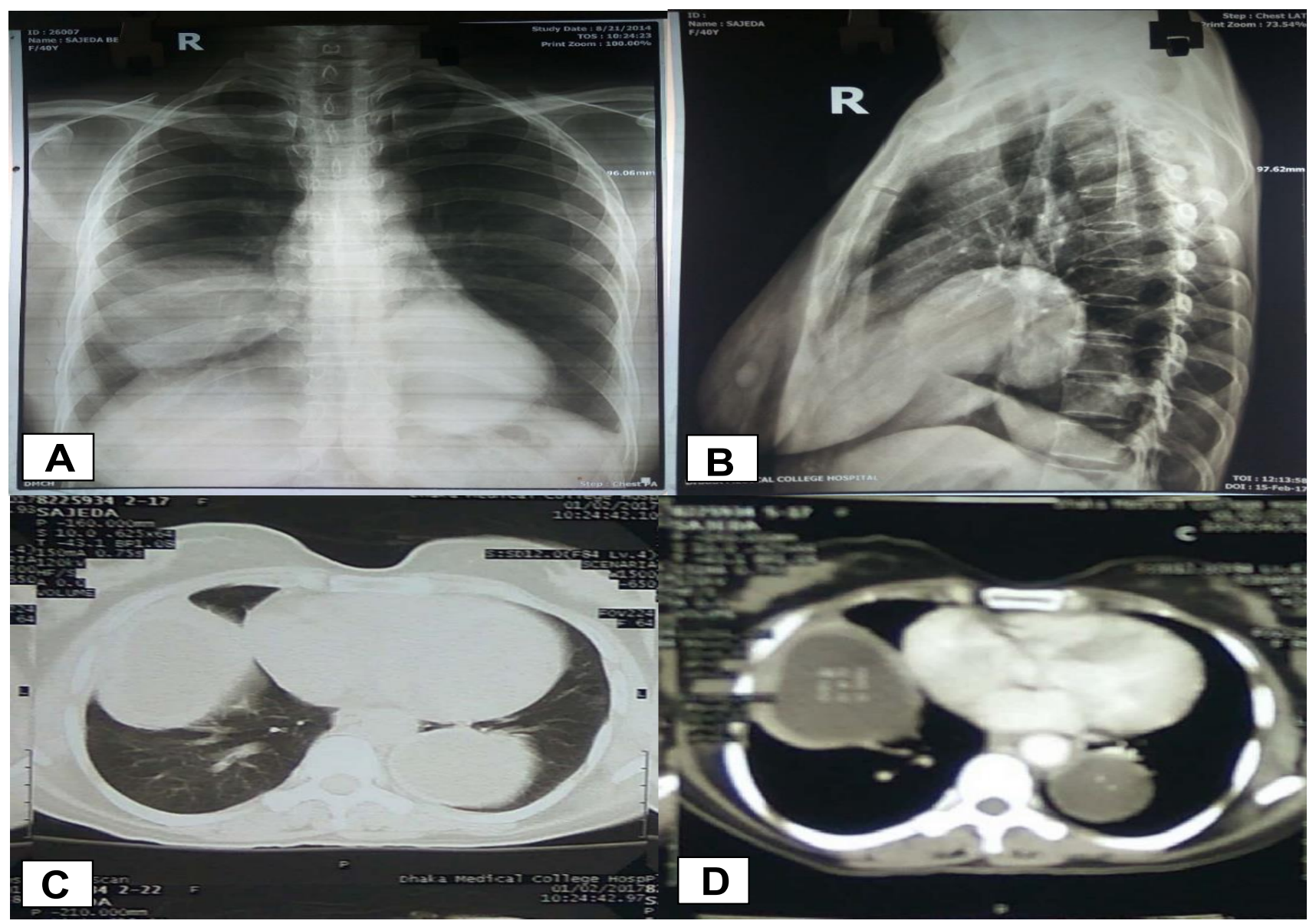

Figure 2: Chest X-ray P/A view A) right lateral view; B) Computed Tomography (CT) scans of chest of the patient before the first operation, Axial slices; C) Pulmonary window view and D) I/V Contrast Mediastinal view showing bilateral pulmonary hydatid cysts.

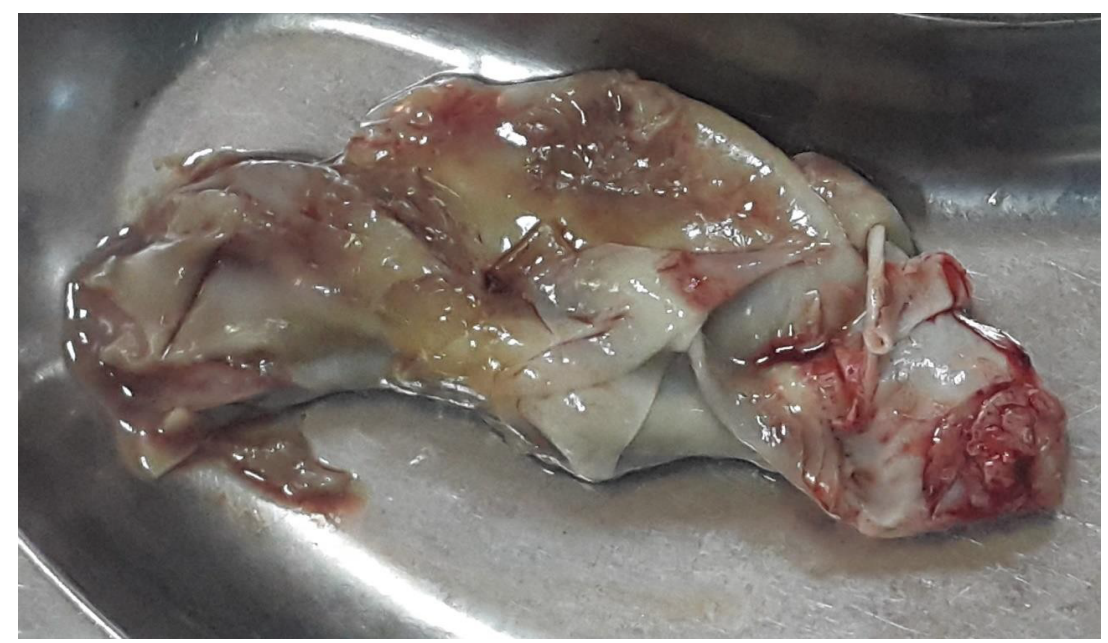

Figure 3: Resected pathological specimen of ruptured and infected pulmonary hydatid cyst of the same patient collected by enucleation of the cyst.

After proper preoperative optimization and preparation with proper counseling and informed written consent, she was planned for VATS enucleation of the pulmonary hydatid cyst (left) but due to excessive adhesion and difficulty in localization of hydatid cyst by VATS, the procedure was converted to minimally invasive thoracotomy and enucleation of pulmo- nary hydatid cyst (left) with capitonnage of the residual cavity was done. The post-operative period was uneventful except she developed surgical emphysema with persistent air leak on $2^{\text {nd }}$ post-operative day which was treated accordingly and subsided by $8^{\text {th }}$ postoperative day. The histopathology report of resected pathological specimen (Figure 3) revealed outer 
fibrous laminar and inner germinal layer of the cyst wall containing brood capsules with scolices, compatible with Hydatid Cyst. She was discharged with proper treatment and advices on $11^{\text {th }}$ post-operative day. After 3 weeks of operation, she came for follow up and her physical examination and chest $\mathrm{X}$-ray revealed normal findings.

\section{Discussion}

Human echinococcosis is a parasitic zoonotic disease that is distributed worldwide, caused by the genus Echinococcus [1]. Carnivores are definitive hosts of the parasite; Humans are accidental intermediate hosts and harbor cysts but unable to transmit the disease. Humans may be infected either through the ingestion of food or fluids contaminated with the parasites eggs shed in the faeces of the definitive host or by hand-to-mouth transfer of eggs after direct contact with thecontaminated fur of a definitive host which transformed into larvae and involve various organs of the body via hematogenous and lymphatic routes $[6,7]$. The Pulmonary Hydatid cyst may remain asymptomatic for a long time [7]. Rupture of the cyst may occur in about $49 \%$ of the cases. After the cyst rupture, patients may present with sudden onset of chest pain, fever, and hemoptysis. The nature of the ruptures viz. contained, communicating and direct, determine the clinical presentation of complicated cysts [3]. The degeneration of the cyst wall causes rupture of the cyst into the bronchus and develop productive cough with huge amount of salty mucoid sputum, cyst fluid and sometimes laminated cystic membrane $[7,8]$. These features are compatible with our case. The pulmonary hydatidosis may be evaluated with some investigations where the radiological signs are more specific to the disease than clinical presentation. Initially, the case may be evaluated with Chest X-ray. Typical CXR finding of uncomplicated pulmonary hydatidosis are single or multiple homogenous round cystic mass with smooth borders. Radiologically, pulmonary hydatid cyst may show some characteristic signs like crescent or meniscus sign, water lily sign, combos sign, camelotte sign etc [7].

The chest $\mathrm{X}$-ray findings may change following rupture of the cyst which may demand further evaluation by Computed Tomography (CT) imaging. The signs "water lily sign" or "floating lily sign" may also be seen in CT scan [8]. The contrast-enhanced chest CT-scan may demonstrate a thin cyst wall with enhancement and the presence of daughter cysts, septation or water lily sign in ruptured cyst confirms the diagnosis [7]. In our case, first CT scan of chest showed bilateral hydatidosis with enhancement of the cyst wall without daughter cysts. And in our second chest CT scan showed thick asymmetric wall with septation and air fluid level in left lower lobe of lung.

Routine blood tests are generally nonspecific in pulmonary hydatidosis and leakage of antigenic substance may result eosinophilia in only $15 \%$ of patients [7]. In our case, serological and immunologic tests were negative. We do not use these tests routinely.

The percutaneous aspiration of cyst can establish the diagnosis of hydatidosis by demonstrating the protoscolices, hook lets or laminated membranes, but it is not rec- ommended as routine diagnostic tool because spillage of content leads to lethal anaphylaxis [7]. The percutaneous aspiration for diagnosis of hydatid cyst is not a diagnostic approach in our area.

Medical treatment of Hydatid cyst may be considered with anthelminthic like Albendazole and Praziquantel. However, we do not follow this protocol. The treatment of Hydatid cyst is principally surgical excision or enucleation of the cyst with removal of the endocyst, avoidance of contamination and management of the residual cavity by capitonnage or marsupialization, with maximum preservation of normal lung parenchyma. The spread of daughter cyst if present and anaphylactic reaction may be prevented by use of scolicidal agents such as hypertonic saline or $0.5 \%$ silver nitrate solutions before opening the cavities $[2,7,8]$. We followed the same principles for the management of our patient.

Definitive diagnosis can be made by extraction of laminated membranes or histologically by demonstration of parasite in excised tissue [7]. Likewise, in our case, peroperative findings and histopathology examination of the resected specimen provided the definitive diagnosis.

\section{Conclusion}

Bilateral Pulmonary Hydatid Cysts complicated by rupture and infection is a rare entity. Although some of the cases of pulmonary hydatidosis may remain asymptomatic, the patients with cough, fever, chest pain and hemoptysis should alert the treating doctor to keep the disease in the differential diagnoses. The respiratory physician and thoracic surgeons should have sound knowledge of this uncommon disease for quick management, as the unfortunate rupture of the hydatid cyst is associated with fatal complication i.e. anaphylactic reaction.

\section{Conflict of Interest}

The authors have no conflicts of interest to declare.

\section{Acknowledgements}

None.

\section{Place of Study}

Department of Thoracic Surgery, Dhaka Medical College and Hospital, Dhaka, Bangladesh.

\section{References}

1. https://www.who.int/health-topics/echinococcosis/\#tab=tab_1

2. Basavana GH, Siddesh G, Jayaraj BS, et al. (2007) Ruptured Hydatid cyst of lung. J Assoc Physicians India 55: 141-145.

3. Kaur M, Singh R (2013) Ruptured pulmonary hydatid cyst: The camalote sign. Indian J Clin Pract 23: 856-858.

4. Marashi S, Hosseini VS, Saliminia A, et al. (2014) Anaphylactic shock during pulmonary hydatid cyst surgery. Anesth Pain Med 4: e16725.

5. Emlik D, Ödev K, Poyraz N, et al. (2015) Radiological characteristics of pulmonary hydatid cysts. Current Topics in Echinococcosis 8: 137-156. 
6. Aghajanzadeh M, Alavi A, Jafarnegad A, et al. (2017) Multiple and bilaterally pulmonary hydatid cyst mimicking metastatic lesions. J Liver Clin Res 4: 1040.

7. Khurana MS, Narang GS, Ded KS, et al. (2012) Ruptured hydatid cyst of lung. Curr Pediatr Res 16: 156-158.
8. Ekim M, Ekim H (2017) Diagnosis and Treatment of Pulmonary Hydatid Disease. J Liver Clin Res 4: 1038.

DOI: $10.36959 / 582 / 422$ 